\title{
PAOLO CIANFRONE
}

\author{
IL LINGUAGGIO FILMICO ED ARTISTICO \\ NEL CINEMA DI ANTONIONI, \\ I SUOI PARADOSSI E LO SPAZIO DELLA CRITICA
}

Io ho molta paura del pubblico, e anche dei critici. Vorrei poterli prevenire, spiegar loro una quantità di cose prima di lasciarli andare a vedere un mio film. Non ricordo chi ha detto che i libri (quindi anche i film, aggiungo io) dovrebbero essere giudicati da un magistrato e davanti ad una giuria, come dei crimini, e si dovrebbero ascoltare l'accusa e la difesa. (Antonioni 1994, 16)

La chiusura di uno degli scritti più significativi di Antonioni "Fare un film è per me vivere," uscito alla fine degli anni 50 , assume questo tono di sconsolata incomprensione. Le ragioni dell'autore e quelle dello spettatore o del critico raramente si incontrano, quasi mai sono in grado di ascoltarsi,di cogliere il pensiero dell'uno o dell'altro. Forse proprio perché percorrono strade diverse manca loro uno spazio comune, un luogo del dialogo. Il risultato sembra essere una sorta di incomunicabilità tra l'autore e la critica. Questo tema, che è quasi un sinonimo per Antonioni, è così pervasivo da non riguardare piu' semplicemente la sua opera, il modo in cui i personaggi vengono rappresentati, ma anche il modo in cui questi vengono recepiti, letti e osservati. Il cortocircuito del sistema dei personaggi si riproduce al livello della fruizione dello spettatore e del critico.

In quello scritto Antonioni voleva polemicamente parafrasare Flaubert. Come questi sosteneva che vivere non era il suo "mestiere," bensì lo scrivere, per Antonioni, al contrario, fare un film implicava il vivere, ne esprimeva le "possibilità." La sua storia personale, egli diceva, "non s'interrompe durante le riprese di un film, anzi è allora che diventa più intensa (ivi, 14). L'opera colta al suo interno, dal punto di vista del suo autore, rivela in realtà un percorso accidentato fatto di "errori, di dubbi, di peccati." La similitudine tra le immagini filmiche nel loro articolarsi e le forme del pensiero indica come le prime siano testmonianza di una processualità. Ciò che un film esprime non è documento di un pensiero, per così clire, fatto compiuto, in sè conchiuso, piuttosto "di un pensiero che si fa"' (ivi, 57). L'autore, il farsi delle immagini, la loro rappresentazione avvengono su un piano di osmosi, 
di reciproca trasmutazione all'interno di una relazione che è il nucleo dell'opera stessa.

Lo spazio della critica, il modo in cui recepisce, classifica e cataloga il testo filmico è assunto da Antonioni quale luogo di un delitto. Il tribunale, nonostante il carattere fittizio delle sue ricostruzioni, appare l'unico terreno possibile in grado di ripristinare le ragioni dellopera e le intenzioni del suo autore. In Antonioni vi è un costante slittamento del piano del senso, rispetto alla lettura possibile clella critica. Le immagini più che suscitare un livello cognitivo dovrebbero indurre all'empatia. Leggendo tra le righe dei suoi scritti sul cinema vi è una costante indicazione, un'esortazione a sentire più che a capire un film (ivi, 79). L'interrogazione stessa attorno al cinema, alle sue immagini postula ununivocità di senso, un sistema di certezze e chiusure che il suo sguardo sul mondo costituzionalmente non possiede. La domanda implica infatti il rimando al livello della ragione laddove questa è spesso incapace a cogliere e rispondere alle ragioni della vita. Il lavoro del suo cinema è topologicamente posto ad un livello inferiore, laddove lo sguardo, per così dire, sente. "Che cosa avviene," si domandava Blanchot, "quando ciò che si vede benché a distanza, sembra toccarvi con un contatto penetrante, quando la maniera di vedere è una sorta di tatto, quando vedere è un 'contatto' a distanza” (Blanchot, 17-18).

L'immagine di Antonioni è, in questo senso, sinestesica; lavora sullo statuto degli scambi percettivi. La ragione, più che comprendersi, vuole sentirsi, collocarsi negli strati più inconsapevoli dell’inclividuo, quelli privi di parola, che non si possono dire ma solo mostrare attraverso le immagini. Eppure nelle formule concise della critica il cinema di Antonioni appare sotto una cliversa luce. Brunetta fa inconsciamente propria l'accusa di Antonioni ed inmagina il suo cinema proprio come un'aula di tribunale. "Il processo istruttorio ha visto sfilare un enorme quantità di testimoni. I capi d'accusa per dimostrare lá situazione di inesorabile desertificazione dei sentimenti e della vita dell'uomo ci sono tutti" (Brunetta 1991b, 557). Sembra cli essere dinanzi ad un pirandelliano scambio delle parti clove la difesa vede la sua parola, tramutata in accusa. Eppure Antonioni ha pervicacemente protestato contro tutto ciò. Nell'equiparazione tra la sua opera e quella di Pavese e nel presunto pessimismo, quale loro denominatore comune, la sua risposta è chiaral.

Credo che per Parese lesperienza intellettuale abbia coinciso tragicamente con le sue esperienze personali. Si può dire altrettanto di me? Non sto fácendo film clirei quasi con accanimento? E questo accanimento, dopotuto, non potreble essere letto come una prova di ottimismo? (Antonioni 1994, 128) 
Come mai avviene questo iato tra ragioni dellautore e clella critica? Come mai questa sordita per le parole dellatutore stesso? Non potreble forse essere clue gli apparali critici, nel momento in cui pongono le loro clomande, postulano le risposte stesse e che ogni impianto concettuale, come un filtro, non fa che far affiorare ciò che è contenuto nelle sue stesse premesse? Quando Deleuze parla dellarte di Antonioni egli trova "uno sfruttamento sorprenclente dei tempi morti della banalità quotidiana " e "un trattamento clelle situazioni linite che lo spinge fino a paesággi disumanati, a spazi svuotati che si direbbe abbiano assorbito i personaggi e le azioni per conservarne solo una descrizione geofisica, un inventario astratto" (Deleuze, 15).

Antonioni, seconclo questa prospettiva, distillerebbe la realtà nei suoi elementi geometrici. Di un fatto, delle persone o cose che lo costituiscono, non rimangono che relazioni disincarnate, una sorta di quadro geometrico. Richiamandosi alla dicotomia proposta all'inizio del secolo da Worringer che intravvedeva nell'arte due diversi percorsi, quello dellastrazione, da una parte e quello dell'empatia clall'altra, Deleuze sostiene che Antonioni è un tipico rappresentante di un rapporto volto allastrazione. ad una critica oggettiva del reale. (ivi, 15-6). Pensando a ciò che Antonioni diceva a proposito di Deserto rosso, "un film che va forse più sentito che capito" (Antonioni 1994, 79), sembra di trovarsi su un crinale opposto. Un invito all'empatia da parte dell'autore, un'indicazione di astrazione cla parte della critica. Parlare di critica oggettiva al reale implica il rimando ad un apparato concettuale. Ora le tradizionali categorie di soggetto e oggetto sono ancora applicabili al cinema di Antonioni? Qui la lingua stessa, costituzionalmente portata a ragionare nei termini di soggetto e predicato,potrebbe divenire un ostacolo. La natura dellimmagine sembra infatti far emergere una differente grammatica. Bisognerà muoversi con la consapevolezza che i temini propri della lingua non necessariamente si riflettono specularmente nella natura dell'immagine.

Di là da queste possibili differenze categoriali la critica neorealista postula un lessico classico con il quale è necessario il confronto. In questo senso il realismo classico presuppone una rappresentabilità delloggetto realtà che pare avere una sua autonoma consistenza. L'autore tanto più sarà in grado cli raffigurare il reale, quanto più come soggetto si risolverà in esso. Nessuna immaginazione deve prendere il luogo della realtà, sostituirsi ad essa. Loggetto realtà diviene immagine che pare essersi fatta da sè. Le dilficoltà di questo realismo ingenuo mostrano però come la negazione del soggetto porti con sè una sua snisurata affermazione. Egli è come un deus absconditus presente ovunque ma non visibile in alcun luogo. 
Ora Antonioni e buona parte della poetica neorealista partono dal presupposto che la realtà sia indistinguibile dal soggetto che la rappresenta. Un fatto è normalmente qualcosa che accade in un certo spazio e in un dato tempo. Delle relazioni necessarie legano la dimensione temporale a quella spaziale e dicono che certe persone o cose hanno assunto una data configurazione in un certo tempo. Nell'evento vi è come una necessità interna alle cose, nella visione, invece, qualcosa inerisce al soggetto. "Vedere," dice Antonioni, "per 'noi' è una necessità" (ivi, 50). L' uso del pronome indica che la trama dell'accadimento, la tensione nel rapporto è qualcosa che avviene tra l'oggetto raffigurato e colui che lo raffigura. Antonioni ricorda, a questo proposito, un fatto avvenuto a Nizza, durante la seconda guerra, mentre aspettava di raggiungere a Parigi M. Carnè. Il cielo bianco, il lungomare deserto, gli alberghi semichiusi, sulla spiaggia non c'è nessuno, tranne un bagnante che fa il morto. Una ragazza si dirige verso il mare. Un urlo secco, breve. Il bagnante non fa il morto, è morto. In pochi minuti, mentre il bagnino tenta la respirazione artificiale, la spiaggia si riempie di gente. Ora la traduzione in immagine di tutto ciò avviene attraverso un paradosso. "Per prima cosa," dice Antonioni, "io proverei a togliere il fatto" (ivi, 58). Quello che a lui interessa è, per così dire, l'antefatto. Il cielo bianco, la figura solitaria, il silenzio hanno una forza di per sè. A questo insieme il fatto non aggiunge nulla. Potremmo dire, usando una configurazione pittorica, che alla figura, alla sua significatività Antonioni voglia sostituire lo sfondo. Questo non significa che l'uno neghi l'altra, piuttosto che l'uno porti a compimento l'altra. Ma lo sfondo, l'assenza di figura soggettiva, quella sorta di pura oggettività geometrica, in realtà è tesa all'altro da sè, ovvero al tentativo di obbiettivare gli stati d’animo del soggetto.

L'antefatto allora, più che costituirsi in forma astratta dello spazio, vuole tentare d'essere rappresentazione sensibile, attraverso immagini esterne, di ciò che il soggetto prova e sente. "Il vuoto vero," dice Antonioni, "il malessere, l'angoscia, la nausée, il letargo di tutti i sentimenti e desideri legittimi, la paura, la rabbia io li riprovai quando.....mi trovai in quel bianco, in quel niente che prendeva forma intorno ad un punto nero" (ibidem). Qui in questa enfatizzazione degli stati delle cose si sarebbe tentati a porre un accostamento a Robbe-Grillet.eppure il rimando pare debole. Non solo per ciò che Antonioni stesso letteralmente affermava.

Devo dire onestamente che quando i francesi cominciarono a parlare dell'École dn regard a proposito dei miei film, non avevo letto nessun nontean roman. Li ho letti dopo. Ho sempre dato molta importanza agli oggetti. Un oggetto può avere nelle inquadratura alterettanta importanza che una per- 
sona. L'importante 2 far si che uno spettatore si impossessi degli oggetti come dei personaggi. (ivi, 188 )

In Robbe-Grillet la trascrizione del reale avviene attraverso un processo di radicale spersonalizzazione. In "La1 spiaggia" (Robbe-Grillet, $45-50$ ) lo scenario è identico a quello di Antonioni ma il carattere di istantaneita degli statti di cose descritti è programmaticamente privo di raccordi; non vi è alcun senso soggettivo che stia lì a correlarli. Quella sorta di equivalenza trá l'individuo e il mondo delle cose, per cui l'uno trapassa nell'altro, che è lá caratteristca del mondo di Antonioni, in Robbe-Grillet è assente. Se è vero clue assistiamo ad una nuova topologia dello spazio questa non arriva mai, in Antonioni, sino ad una radicale negazione di antropocentrismo (cfr. Brunetta 1991a, 553). Se gli oggetti, gli stati di cose, nella loro immobilità creano, da un lato, un senso di perturbante straniamento, dallaltra essi possono essere letti come precipitato sociale. La loro irriclucibilità, il fatto che l'inquadratura non riesca a piegare del tutto la loro naturale clurezza, è positivamente da leggere quale segno sociale. Al perturbante degli oggetti risponde il loro essere residuo sociale, se si vuole, grammatica (cfr. Adorno. 82). Questo trasmutare tra mondo delle cose e delle persone, se toglie fondamento a qualsivoglia poetica realista, mostrandone il carattere illusorio, toglie altresì possibilità all'opzione oppostá. Lá possibilità solipsistica, il mondo è il mio mondo, per Antonioni non si da. Luso del possessivo rimanda ad un io pieno, padrone di sè, e della scena, che è invece quanto qui si tenta continuamente di mettere in discussione, di stuotare, scompaginare. È l'uscire da sè, dal proprio vuoto, il tragitto da compiere. Il problema è, nelle sue parole, "intonare i dati clella nostra personale esperienza con quelli cli un'esperienza più generale, così come, secondo Antonioni, il tempo individuale si accorcla a quello cosmico" (ivi, 51). Il soggetto che lega il suo sentire a ciò che lo oltrepassá, alla dimensione della comunità a cui appartiene e che coniuga il suo tempo a quello del mondo di cui fá parte, continua a riproporre la sua centralità seppur entro precarie coordinate. Conseguenza di questa posizione è che una sortá di principio di indeterminazione non fa più discernere ciò che nell’immagine è réale da ciò che è immaginazione.

Il problema è che il cinema in Antonioni, non è più al servizio clella realtà, suo strumento riproduttivo. È la realtà che è al servizio di quel cinema (ivi, 61). nel senso che la sua finzione è il miglior modo per estrarre possibili significati dal reale. Citando Wittgenstein, Antonioni sostiene che "l'immagine ha la forma di una realta che non esiste "(Tinazzi. XXVIII). Qui l’immagine è forma, configurazione. Essa, per sua natura, lavora tra domini diversi tra- 
ducendo e trasportando elementi da un dominio all'altro. Wittgenstein cercando di esemplificare tale problema ci ricorda che la relazione tra una proposizione quale "il lasciare la stanza" e l' "immagine" di ciò che un ragazzo fa se obbedisce ad un tale ordine la si può paragonare al fatto matematico che $2+3$ è realmente un'immagine di 5 (cfr. Wittgenstein 1993, 57). Ma l'immagine, nel suo tradurre piani 'diversi' postula che il suo essere non abbia una corrisponclente realtà. Da qui l'idea che l'immagine sia sostanzialmente forma, sintassi, non semantica. L'immagine, potremmo dire, è segno, non significato, come la figura della pipa di Magritte "n'est pas une pipe." Il piano delle immagini come segni, nella sua combinatoria, diviene significativo poiché esso riflette le tante possibilità che il dato di fatto contiene. L'immagine è il mondo possibile che è significativo proprio in quanto illumina il reale e ne coglie i suoi significati riposti. In fondo di tutto ciò che è pensabile è possibile farsene un'immagine (Wittgenstein 1964, 3.001).

In questo percorso che prende le distanze dalla mimesis degli accadimenti avviene che la finzione dell'immagine sia in grado, oltrepassando i fatti, di comprenderli a loro volta. Proviamo a capire meglio questo. Come può Antonioni sostituire il fatto con l'antefatto senza cancellarne il significato, anzi, come lui sostiene, comprendendolo appieno. È che l'antefatto oggettivo, divenendo soggetto lo assimila a sè, e così facendo, ne estrae le sue possibilità. La storia non è più mero accádere, fatto bruto, ma diviene storia che comprende se stessal e che per libertà di soluzioni e per intensità supera ed invera sè (Antonioni 1964, XIII). Vi è dunque un paradosso all'interno del cinema di Antonioni. Come nel film a soggetto il personaggio deve riflettere l'ordinarietà del reale, má al contempo, se si vuole, la sua idealità, quasi che la direzione del suo agire si accordasse "irrazionalmente con le direzioni altrui."

Il personaggio è, per così clire, al contempo dentro di sè, nel mondo delle cose e fuori di sè, in sintonia con qualcosa che lo oltrepassa. In questa zona di confine, di compresenza paradossale, l'autentico sono i suoi "errori ." "Lasciarlo sbagliare," dice Antonioni, "perché i suoi errori sono quanto di più spontaneo egli possa offrire in quel momento" (ivi, 49). Così, appunto, dato essenziale sarà l'umiltà, l'unica in grado di "superare il limite della sua stessa natura" (ivi ). Essere in sè ed oltre sè, solo così si arriva ad "un significato che comprende anche la storia raccontata." Il classico tema dell'allegoria nel cinema e dunque del personaggio allegorico, se si vuole del modo in cui un immagine rappresenta una realtà differente rispetto alla sua 'lettera' per Antonioni è tutto qui, ma modernamente esso si da non nella traparenza concettuale di questo procedimento, nella chiara iclentificazione del per- 
corso retorico, piuttosto nellat problemalticità paradosisale.

E significativo che egli volesse sceneggiare i primi capitoli della Imboduzione alla filosofia della matematica di B. Russell. È noto come Russell. proprio parlando dell'insieme cle contiene se stesso abloia demolito tutti i tentativi di fondazione della matematica che risalivano alla fine del secolo scorso. I'n individuo, potremmo dire, non può al tempo stesso essere se stesso e clisse. "Il numero 3," dice Antonioni, "non è identilicabile col terzetto composto dai signori Brown, Jones e Robinson. Il numero 3 è clualcosal che tutti i terzetti hanno in comune"(Antonioni 196\%, XIII). Egli qui gioca sul contrasto tra la serietà logica e. come egli dice, la comicità della traduzione, il terzetto dei signori che si colora di ridicolo.

Ma l'umorismo qui si inscrive all'interno dei rapporti tra figura es sfondo. Ciò che sono semplici elementi della classe. ordinari attori che calcano la scena, non possono assurgere ad essere totalità della scena, se si vuole, della classe. se non in modo appunto comico. La comicità sta in questa compresenza tra la corporeità del terzetto e l’immaterialità clella classe, che non può risolversi nei 3 personaggi che divengono goffi proprio in questa pretesa di totalità. má può però oscillare in loro. Così quando si abita questo andirivieni tra figura e sfondo, si è nel paradosso dell umorismo, nella sua terra di confine tra domini cliversi. Qualcosa è sè, ma al contempo altro da sè. individuo e classe. senza risolversi mai compiutamente né nell'uno, né nell'altro. Cosi la resa geometrico-matematica del reale, in Antonioni, non si appiattisce sul versante di una vuota e desolata astrazione. Questa è solo un aspetto apparente. Al contrario laddore questa supposta astrazione si da, si è come in presenza di qualcosa di palpitante, quella zoná nelláa quale esperienze opposte si cercano, di là da qualsiasi criterio logico.

Ĺno dei derivati dei paradossi di Antonioni sta in questa oscillazione tra figura e sfondo. Noi qui potremmo quasi ricostruire alcuni tasselli cli una sua pinacoteca ideale. Uno di questi è certamente costituito cla Matisse ( cfr. Brunetta 1991b, 20 ). E lorse paradigmatico ricordare l'incontro di Antonioni con lui. Doveva fargli un'intervista. Aspettò senzesito nel suo studio. Nell'attesa scriveva, stendevá appunti. Il risultato, comegegli ricordál, è stata "un'intervista a Matisse senza Matisse" (Antonioni 1994, 221), un incontro senza incontro, un paradosso ancora. Ma il Matisse che a lui interessa è quello, per cosi dire, orientalista dore la figura, il primo piano. viene per cosi dire fagocitato dallo sfondo arabescato sul quale è posto. Nelle parole di Matisse: "Lá rivelazione mi è venuta calloriente" (Matisse, 23). Dore inizia la figura e dove finisce lo sfondo è posto ad un livello di indiscernibilità che non è dato risolvere. Importante qui non è la giustapposizione degli ele- 
menti, la loro tessitura per così dire paratattica o la loro distinguibilità categoriale, l'essere appunto l'uno soggetto e l'altro oggetto, quanto invece il loro carattere relazionale. In Matisse e nell'estetica orientale che ne è alla base, si assiste ad un processo rituale di trasformazione. La con-fusione tra soggetto e oggetto, tra figura e sfondo allude all'arte come ad un'esperienza di trasformazione (cfr. Duthuit, 1998, 323 e seg.). È forse un caso, allora , che la costante del cinema di Antonioni si basi sulla suggestione del viaggio, che è avventura, ripercorso, fuga, ricerca di identità (Tinazzi, op. cit., XIX). Il viaggio, questa figura che si clispone sul crinale dello spazio esterno allude in realtà significativamente a quello interno, quale luogo della trasformazione, della possibile agnizione.

L'an'ventura segna l'inizio di questo rapporto con il viaggio, il raffigurare personaggi colti nei loro movimenti nello spazio. L'isola è immagine di un territorio separato dove il tempo scorre ma in una sorte di sospensione, clove il mare allontana e circoscrive $\mathrm{i}$ personaggi in un luogo chiuso nel quale inscrivere le loro azioni entro forme labirintiche. La lettura di questo materiale filmico sembra quella di uno spazio recluso, di una prigione, luogo dell'impossibilità, delle domande ossessive vissute come coazione a ripetere intorno alla gracilità dei sentimenti o all'inconoscibilità del reale (Di Giammatteo, 235 e seg.). La forma labirintica non ha però necessariamente questa portata negativa. Essa è storicamente il luogo del peregrinare, lo spázio di una ricerca. Si tratta di por mano al disordine dei sentimenti, al loro casuale clisporsi, a quella sensazione di ordine inafferrabile. Si potrebbe alloral dire che il labirinto, prima che nelle cose, nelle azioni, nei personaggi che lo incarnano è clentro l'autore stesso. Entrare nella sua opera assume le forme del labirinto, rimanda ai passaggi, ai corridoi,alle porte cli cui il testo filmico è costituito.Le cliverse scene, gli spazi aperti e chiusi hanno questo ritmo che non è altro che un richiamo alla relazione tra disordine $e$ creazione. Il labirinto allora è positivamente forma d'ordine che si impone a ciò che è indistinto, spazio della riorganizzazione e della ricerca. In questo senso il testo filmico isomorficamente riflette il suo farsi assumendo le vesti di uno specchio visibile che riproduce ciò che invisibilmente avviene clentro l'autore e la sua opera. L'aucentura parla sì dei personaggi ma, al tempo stesso, parla del suo autore. Avventura esterna ed interna, luoghi dell'azione e della creazione non sono sempre distinguibili.

Sul piano delle dinamiche interne unaltra figura risulta fondamentale. Nelle parole di Antonioni che riflettono il comporsi del testo filmico il problema era "come raccontare le verità del film e intanto far tacere le altre che pullulavano ai margini, che premevano con tanta forza? Assumere le une 
come misure delle altre?"( Antonioni 199+, 77). Ancora una volta la figura e lo sfondo è ciò che muove dietro le quinte la sua opera, il margine che cliviene centro e il centro margine. Le stesse nozioni tecniche del suo cinemá vamno rivista a partire clá questotticá. La perclita di centralità clella figura in Antonioni, nella sua traduzione filmica, viene ad assumere il nome di décadrage, orvero decentratura. (Aumont, 84). Si trattá di creare un vuoto al centro dell'immagine, laddove il cinema tende classicamente a riempire. E se lo sfondo, la bordurá dell'immagine viene, per così dire, tralasciata, qui, al contrario, essa è accentuata. Ma il vuoto non allude ad un'esperienza di pura negazione, quanto di affermazione. I personaggi spariscono sì, ma per meglio affermarsi sotto le mutate spoglie del paesaggio sfondo. Il vuoto è, allora, propriamente un pieno; l'assenza non allude che a presenza. Se la centratura è rispetto clella scenicità dell'immagine e non della realtà, il décadrage non necessariamente é "lacuna destinata a non risolversi mai, perpetua tensione di un corpo che mai si riconcilia con lo spazio" (ivi, 109), quanto luogo-relazione nel quale il corpo compie il suo viaggio-ritrovamento nello spazio. Non vi è propriamente sguardo indietro verso il passato, una sorta di nostalgia per qualcosa che se mai è stato, ora non è più (ibidem), piuttosto una proiezione in avanti, utopica, verso qualcosa che potrebbe essere. Come l'immagine è la levatrice del possibile che il reale contiene quale sua mera possibilità, così la decentratura, il vuoto che prende il sopravvento non ha una valenza meramente negativa, semmai propositiva, incarnando la tensione ad oltrepassare, verso qualcosa che non è ancora ma che potrebbe essere. È appunto lo spirito dell'utopista che Barthes ritrova in Antonioni (in Zemignan, 8).

Noi potremmo rileggere i paraclossi in Antonioni come un'insufficienza dei criteri classici di verità e, al contempo, come un'apertura alle implicazioni relative al senso delle espressioni. Se una proposizione è, relativamente al suo significato, inscrivibile all'interno della rigida alternativa di vero o falso, per quanto riguarda il senso invece, essa si muove all'interno degli infiniti modi che noi abbiamo per circoscrivere quel medesimo significato. Uno stesso oggetto può essere raffigurato attraverso differenti espressioni che incarnano così i suoi diversi sensi. Ora Antonioni, di là clai criteri logici cli verità, si muove con partecipazione sul piano del senso. Egli è interessato non tanto agli aspetti denotativi del reale, quanto connotativi, ovvero ai modi possibili attraverso i quali il reale si fa immagine, diviene visione. Come l'attore proprio nel momento in cui sbaglia, in quell'atto involontario allarga gli spazi di esperienza, cosi le sostituzioni dei momenti secondari alla scena madre, tipiche del cinema di Antonioni, (Antonioni I994, 24) vanno 
nella direzione di scompaginare i modelli tradizionali e di allargare gli spazi di vita.

S. Chatman (in Zemignan, 37 e seg.) legge le possibilità connotative di Antonioni sul versante letterario. Se la letteratura del ventesimo secolo, da Joyce a Proust, è priva di sequenzialità logica, è de-narrativizzata, tanto che in essa non pare accadere assolutamente nulla, allo stesso modo in Antonioni il legame tra gli eventi è privo di causa. Gli accadimenti importanti, il centro della scena non risultano elementi significativi, rilevante in una lettura sequenziale è l'intreccio, lo svilupparsi degli eventi, poiché dell'evento, del fatto decisiva è la condizione della sua possibilità, l'antefatto appunto. Qui spesso le condizioni più che causali risultano casuali. Se la causalità si gioca nelle maglie strette della logica, dove ben poca esperienza passa, poca vita reale lì conosce esperienza, la casualità diversamente volge alla divagazione, alla contemplazione lenta per le minute cose. Non più in grado di decidere su quale sia il loro effettivo statuto, se figura o sfondo, esse vengono rese, tramite l'ellissi, come una traduzione visiva di tempi morti "che ritraggono ancora a lungo la scena, dopo che gli attori se ne sono andati." Attraverso la manipolazione ellitticá dell'immagine si ha logicamente la difficoltà di esperire i nessi causali e, sul piano del soggetto, l'impossibilità di rendere conto, in modo definitivo, delle sue emozioni. Ma è bene dire che la lingua si da nel tempo, nella successione delle sue lettere e il suo senso si coglie solo alla fine, mentre l'immagine è pertinenza dello spazio.

La lettura puramente letteraria di Chatman pare non rendere del tutto conto dei caratteri tipici dello spazio visivo. In Deserto rosso ma anche in Blou' up o in Zabriskie Point, nelle scene di chiusura di questi film è sempre to sfondo a parlare, non un personaggio. Il rapporto che la narrativa tende ad istituire tra la narrazione di un soggetto e la descrizione di un oggetto viene qui rovesciato. Una sorta di estetica orientale è alla base di questi processi di reversibilità dove lo sfondo-oggetto diviene non tanto approdo nichilistico, il nulla che conclude la vicenda, piuttosto soggetto che la continua, seppur sotto differenti spoglie ed in modo aperto. Un approdo vero non vi è mai, semmai continua tensione, ricerca. L'orizzonte di Antonioni vive all interno di una permutazione costante delle categorie. Ora l'arabesco si colloca proprio in una zona di confine tra testo scritto e spazio pittorico. Se quest'ultimo si coglie immediatamente nello spazio che lo costituisce, quello si da nel tempo del suo discorso. L'arabesco invece pertiene allo spazio pittorico ma, al medesimo istante, si legge nel tempo del testo. È il luogo per eccellenza delle trasformazioni, delle metamorfosi. Lì avvengono tutti gli scambi tra figura e sfonclo, lì il vuoto diventa pieno e il pieno 
vuoto. Allora è nel Matisse orientale che l'immagine trova le condizioni di un empo che si da nello spazio é di uno spazio che è immagine del tempo (cfr. in unal diversil prospettiva S. Bernardi, 150).

In fondo Worringer, richiamando la categoria dellastrazione, voleva andare al di là del classico concetto di mimesis, dellarte come copia fedele della natura che finiva per escludere esperienze artistiche di civilta cliverse da quella europea e cli differenti ma cruciali periodi storici. Egli, in questa attenzione per l'astrazione, voleval rendere conto anche di una parte cospicua dellarte di questo secolo. In Worringer pareva però esserci una qualifica puramente negativa, un senso di angoscia che presiede l'origine delle forme geometriche: l'uomo rispondeva al caos della natura con la regolarità. Ln'operazione di ritiro dal mondo, di sottrazione era, secondo lui. alla base dellastrazione. A questa dimensione se ne aggiunge unaltra che è connessa alla transitorietì della realtà, alla estrema relatività cli tutto ciò che accade. In questa accezione l'astrazione nei popoli orientali non arrebbe un carattere solamente negativo, poiché non è qualcosa che si colloca semplicemente "prima della conoscenza, ma al di sopra della conoscenza" (Worringer.40 e seg.).

Lastrazione, allora. non è un mero ritiro dal mondo. poiché essa potrebbe realizzare l'assunto opposto. Si potrebbe dire, accettando questo lessico, che per Antonioni una visione priva di astrazione è un vedere cieco. laddove un astrazione senza visione è esercizio formale puramente ruoto. Questi due aspetti in Antonioni non si escludono l'un l'altro, piuttosto si includono reciprocamente. Ciò che gli interessa del cinema orientale è "J'orizzonte degli avvenimenti." Se in occidente gli accadimenti si danno sotto il segno della dualità, da un lato l'abbassarsi nell'orizzonte quotidiano dell'uomo e dall'altro l’innalzarsi nell’inaccessibile, per così dire cosmologico, nel cinema giapponese egli ritrova invece un medesimo orizzonte che lega questi due diversi aspetti. Un solo tempo, potremmo dire, rappresenta ciò che muta e l'immutabile (Antonioni 1978,11). L'accidente, l'individuale è al tempo stesso generale, e lo sguardo, proprio in tale capacita di coniugare gli opposti si fa visione. È questo che interessa Antonioni, ma in tale prospettiva non ci si muove più secondo la dicotomia proposta da Worringer.

Quando D.H. Lawrence parla della natura morta. del dipingere un paio di mele, egli dice che per farne un immagine "vivente" il pittore cleve essere "un puro di spirito e. per tutto il tempo in cui è stato immerso nella composizione, suo è stato il regno dei cieli" (Lawrence. vol.11, 822). È un trasumanare allora la condizione creativa dellartista, ritrarre l'al di cua essendo nellàl di là. Ed è questo paradosso che si ritrova nel lavorio costante delle 
tele, per esempio in Cézanne, con la sua continua operazione di svuotamento. La forma dell'immagine non è mimesis del reale, piuttosto sua parodia, cliché. Si tratta di seppellire queste forme, di combatterle con i margini, i contorni e, una volta morto il cliché, seppellire anche il suo fantasma (ivi, 997).

Il gioco tra figura e sfondo è tutto interno a questa nuova grammatica. Puntare sulla reversibilità tra queste due polarità implica andar oltre un ingenuo antropocentrismo. L'individuo continua ad esistere, ma deve mutare il suo modo di guardare, modificare la relazione con sè e con ciò che lo circonda. Antonioni qui rimanda a Wittgenstein, alle difficoltà di vedere ciò che si ha davanti agli occhi (Antonioni 1994, 205). Ogni sguardo tende a ripetere, posto com'è entro un reticolo di classificazioni precostituite. È necessario dimenticare tutto, sostituire l'occhio esterno con quello interno. Vedere dentro (ivi), dice Antonioni, riproponendo ancora l'ennesima permutazione, vedere il mondo nella sua pensabilità, possibilità.

Giocare tra la forma e il margine, tra la figura e lo sfondo, tra il fatto e l'antefatto, destrutturare l'uno nell'altro sono operazioni che rivelano alcune analogie di prospettiva con l'opera di Cézanne riletta da Lawrence e quella di Antonioni. Nei termini di Wittgenstein Antonioni potrebbe dire: "Un'immagine ci teneva prigionieri. E non potevamo venirne fuori perché giaceva nel nostro linguaggio, e questo sembrava ripetercelo inesorabilmente" (Wittgenstein 1967, 115). L'immagine presenta una sorta di coazione a ripetere che bisogna spezzare. Andare allora di là dal reale, lavorando piuttosto sui modi possibili della sua configurazione. In fondo il dominio matematico della nozione di immagine, quale correlazione tra entità diverse, fa pensare che alla dicotomia proposta da Worringer bisognerebbe sostituirne un'altra relativa all'arte del "che cosa" e del "come."

E nello spettro ampio di queste polarità che si possono ricollocare i modi in cui le arti rappresentano (cfr. Arnheim, 67-81). Il che cosa, la configurazione dell'accadimento, lo stato delle cose, e il come, i modi diversi, potenzialmente infiniti attraverso i quali arrivare a quel medesimo stato, non si danno in una modalità di esclusione, quanto di possibile inclusione. È sempre un artista, lo scultore pop americano Oldenburg, a far riflettere Antonioni sulla differenza tra il dire ed il mostrare, tra un'Europa che scrive ed un'America che mostra. (Antonioni 1994, 60). Qui il problema è che solo le arti figurative ed il cinema possono compiutamente mostrare ciò che la scrittura non può dire se non collocandosi sul piano dell'inesprimibile. Che un'immagine giochi sul crinale doppio dell'ordinario e di ciò che lo oltrepassa, dell'essere al tempo stesso, parte di un insieme ed insieme (per rimanere 
ad analogie logiche), è qualcosá che si può solo mostrare, ma non si può clire, dáta l’impossibilità logica. Antonioni è wittgensteiniamente interessato ad esplorare questa zona limite. Se il cinemá è, in quanto tale, teso alláa riproducibilità del reale come sua intrinseca natura (Benjamin, 59), riflettere sul suo linguaggio, sul suo non "clire" ma "mostrare" le cose, porta allá centralità della visione che da mezzo, oggetto di espressione diviene il soggetto stesso del cinema

Qui però non siamo solo in presenza di una pura operazione metalinguistica, autoriflessiva (Tinazzi, XXVII ) poiché positivamente il "mostrare sè," l'esibire la visione è l'unico modo per dire cose altrimenti indicibili. È un leitmotiv, in Antonioni, quello di un cinema che "mostra" immagini. "Non voglio," egli dice, "dimostrare tesi ma "mostrare" storie" (Antonioni 1994, 207). L’immágine, egli sostiene, ha la capacità di annodare il particolare con l'insieme, di nuovo l'individuo con la classe, all'interno di un'oscillazione che prevede distacco e partecipazione (ivi, 218). Ogni spostamento, ogni diversa configurazione delle cose non muta semplicemente lá forma ma il nostro rapporto con le cose. "Il mondo è il concetto che è sempre presente in un "immagine" ( ivi: 188). Questo significa che per Antonioni non vi è nessuna apertura originaria sul mondo. Le immagini sono sempre costruzioni, concettualizzazioni senza le quali il mondo nella sua oggettualità non può clarsi.

Ma una concettualizzazione non può parlare di sè senza incorrere in irrisolvibili paradossi. Vi è una lunga storia che scandisce la logica e i suoi nodi. Le differenze categoriali non si possono esprimere ma solo mostrare. Questa è la risposta di Frege (Frege, 171 e seg.) nel secolo scorso ed Antonioni, ovviamente dal suo proprio versante, pare muoversi su un crinale simile. Un'operazione metalinguistica tencle a parlare di sè, a spiegare sè, come la teoria dei tipi di Russell prevede entrate ed uscite, chiare gerarchie e gradini. Non la confusione dei piani má la loro linda separazione. Parlando a proposito di Blou up film, se si vuole, metalinguistico per eccellenza, dove il mondo è riclotto continuamente ad immagine, alla sua rappresentazione fotografica, egli dice che "è come lo zen: nel momento in cui lo si spiega lo si tradisce" (jvi, 137). Già dal titolo con la sua istanza polisemica che va clall'esplodere, al redarguire, all'ingrandire si capisce come il senso del film sia tutto clentro un orizzonte linguistico. "A che servono i nomi?" dirà il protagonista dinanzi all'aggressione delle ragazze che lo perseguitano in cambio di una foto. di un'immagine. Il nome orviamente non è la persona; tra lingua e mondo vi è un salto, come salto vi è tra il protagonista che ruba immagini e la donna che di queste è clerubata. Alla richiesta delle immagini sottrat- 
te la risposta è: "Almeno dimmi il tuo nome." Il cortocircuito dei piani, egli avrà soltanto un numero di telefono falso, non indica necessariamente che le parole servono solo per ingannare (Di Giammatteo, 331), piuttosto che le parole, ogni parola è priva di purezza, come la vita stessa. Il cadavere che l'ingrandimento dellimmagine prima, la realtà poi, mostra e cancella, il gioco tra figura e sfondo, l'apparente sparizione del protagonista come prima del corpo, non hanno necessariamente una connotazione negativa.

Il problema non è la reversibilita' tra realtà ed illusione, lo scivolare l'una nell'altra, non vi è in tutto ciò un necessario scacco conoscitivo (ivi, 335). Alla domanda se Blou' up sia un film pessimistico, la risposta di Antonioni è spiazzante: "Nient'affatto, perchè alla fine il fotografo ha imparato moltissine cose tra cui giocare con una palla immaginaria" (Antonioni 1994, 137). Il gioco mostra allora il nesso tra immagine e possibile. Vi è come un isomorfismo tra un gioco irreale che svela positivamente lo statuto privo di realta' fattuale proprio delle immagini. Il film dunque "mostra" cose che non si possono dire altrimenti se non tradendole, da qui il suo carattere zen e cosi la lingua, solo dal suo interno può esibire le differenze categoriali che, guardate da fuori, conducono ad inestricabili difficoltà. L'interesse di Antonioni per Pollock nasce da qui. Come il pittore americano ci ricorda : "Io preferisco la tela sul pavimento. Io mi sento più vicino, più una parte del dipinto, dato che in questo modo posso camminare attorno ad esso, lavorare dai 4 lati e letteralmente essere 'nel' dipinto" (in Chipp, 546-8).

Ora di là dalla gratuità del caso, dall'orrore del vuoto e dall'arte che rappresenta sè nell'atto del dipingere, in una sorta di autonomia semantica, di autoriferimento, importante pare essere la scelta topologica del pittore. La rappresentazione non presuppone separazioni, distanze ma sempre appartenenza. L'artista si muove all'interno di un "tessuto" di equivalenze dove ogni distinzione gerarchica, fondamento della rappresentazione prospettica, si e' ormai dissolta. Nessuna "distanza" è più possibile. (cfr. Greenberg, 157). La presenza di Pollock tra i suoi artisti preferiti (Antonioni 1994, 218) consiste proprio in questa mancanza di gerarchie. Il film non è che filtro di un accumulo di immagini nelle quali "rovistare." Le scene chiave sono "brandelli," "frammenti" privi di "curva drammatica." La sceneggiatura non ha alcunché di definitivo, l'improrvisazione, viceversa è, per Antonioni, ciò che detta l'ordine (ivi, 129-3I). L'appartenenza al linguaggio, al suo dominio è qualcosa che caratterizza il farsi del testo filmico. Porsi al di fuori di questo spazio, scegliere la presunta zona franca della critica per spiegarne il senso è qualcosa di improprio.

Per Antonioni infatti, se un film si può spiegare a parole, attraverso la "dis- 
tanza" del linguaggio non e un vero film. Il soggetto e l'oggento, le varie categorizzazioni sin qui usilte. in realtà dal questal prospettiva non hanno alcun senso. Che talj realtà calchino, pere così dire, la scena, non si può dire ma solo mostrále. Il film, come la vita, abita in una zonal di confine, solo accettando la nostra paradossale condizione di abitanti di un luogo, per cosi dire senza luogo, possiamo veclere il suo possibile senso. Dal gioco, direbbe Antonioni, non è possibile uscire. Alla critica rimane forse solo lesortazione di Todorov (Todorov, 183). Non si tratta di parlare dellautore, ma all autore o con l'autore. L'aula del tribunale diviene così lo spazio della párola e dei suoi possibili scambi.

\section{Brock (niceristy}

\section{OPERE CITATE}

Adorno. Theodor W'. Pan'a aesthetica: saggi 1958-196-. Milano: Feltrinelli, 19-9. Antonioni. Michelangelo. Sei film. Torino: Einaudi, 1964. . "Lhorizon des événement," Cabier du cinema, 290 (19-8): 11. Fare un film è per me rizere. Vénezia : Marsilio, 1994.

Arnheim. Rudolf. Intuizione ed intelletto. Milano: Feltrinelli, 198-.

Aumont, Jean J. L'occhio interminabile. Venezia: Marsilio, 1991.

Barthes, Roland. "Caro Antonioni," Il tempo dello sguardo. A cura di R. Zemignan. Padova: I'niversità degli studi, (1991): 7-10.

Benjamin. Walter. Lopera darte nell epoca della sua riproducibilità tecnica. Torino: Einaudi, 1966.

Bernardi, Silvio. Introduzione alla retorica del cinema. Firenze: Le Lettere, 199t.

Blanchot, Maurice. Lo spazio tetterario Torino: Einaudi, 1960.

Brunetta. Gian Piero. "La formazione della poetica di Antonioni e il Neorealismo," Il tempo dello sguardo. A cura di R. Zemignan. Padorà : Lniversità degli Studi.(1991): 19-2-t Cent anni di cinema italiano. Bari:Laterza, 1991.

Chatman, Seymour."Le innovazioni narrative di M. Antonioni," Il tempo dello sguardo. A cura di R. Zenignan. Padora: Lniversità degli Studi.( 1991):3-43.

Chipp, Herschel.B. Theories of Modem Ait. Berkeley: U of California Press, 1968.

Deleuze, Gilles. L'immagine tempo. Nilino: Lbulibri, 1997.

Di Giammatteo. Fernando. Lo sguardo inquieto. Firenze: La Nuova ltalia,199.1.

Duthuit, George. Matisse e lo spazio bizantino. Romna: Artificio. 1998.

Frege, Gottlob. Ricerche logiche. Bologna: Calderini, 19-0.

Greenberg. Clemence. Art and Culture. Boston: Beacon Press, 1961.

Il tempo dello sguardo. A cura di R. Zemignan. Padova: Lniversità degli studi. 1991.

Lawrence, David. 11. Teatro e prose tarie.(volume XT). Milano: Mondadori. 19-5.

Matisse. Henri. "Le chemin de la couleur," Ant present 2 (19.17): 23.

Robbe-Grillet, Alain. Istantanee. Torino: Einaudi. 1963.

Tinazzi, Giorgio. Premessia al M. Antonioni. Fare un film è per me vizere. Venezia: Marsilio, 1994. 
Todorov, Tzvetan. Critica della critica. Torino: Einaudi, 1986.

Wittgenstein, Ludwig. Tractatus. Logico Philosophicus. Torino: Einaudi, 1964. Ricerche filosofiche. Torino: Einaudi 1967.

Philosophical Occasions (1912-1951). Indianapolis Cambridge: Hackett, 1993.

Worringer, Wilhelm. Abstraction and Empatby'. New York: International Universities Press, 1967 